\title{
Expression Analysis of Circulating miR-21 in Iranian Original Article Patients with Hereditary Colorectal Cancer
}

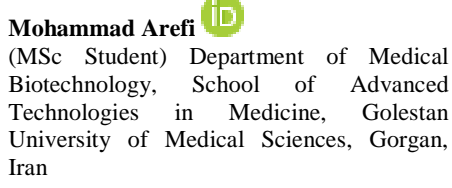

Ayyoob Khosravi

(PhD) Department of Molecular Medicine, School of Advanced Technologies in Medicine, Golestan University of Medical Sciences, Gorgan, Iran

Abdolavahab Moradi

(PhD) Department of Microbiology, School of Medicine, Research Center of Gastroenterology and Hepatology, Golestan University of Medical Sciences, Gorgan,

Seyed Hamid Aghaee-Bakhtiari ID

$(\mathrm{PhD})$ Department of Medical Biotechnology, School of Medicine, Mashhad University of Medical Sciences, Mashhad, Iran

Najmeh Javid ID

$(\mathrm{PhD})$ Department of Microbiology, School of Medicine, Research Center of Gastroenterology and Hepatology, Golestan University of Medical Sciences, Gorgan, Iran

\section{Mehdi Evazalipour}

(PhD) Department of Pharmaceutical Biotechnology, School of Pharmacy, Guilan University of Medical Sciences, Rasht, Iran

\section{Anvarsadat Kianmehr}

(PhD) Department of Medical Biotechnology, School of Advanced Technologies in Medicine, Golestan University of Medical Sciences, Gorgan, Iran and Medical Cellular and Molecular Research Center, Golestan University of Medical Sciences, Gorgan, Iran

Corresponding author: Dr. Anvarsadat Kianmehr

Email: kiabiotpro@yahoo.com

Tel: +989113801426

Address: Department of Medical

Biotechnology, School of Advanced

Technologies in Medicine, Golestan

University of Medical Sciences, Gorgan,

Iran

Received: $2019 / 11 / 05$

Revised: 2019/12/03

Accepted: $2019 / 12 / 14$

\section{(c) (7) \&}

This work is licensed under a Creative Commons Attribution 4.0 License.

DOI: $10.29252 / \mathrm{mlj} .15 .2 .1$

\section{ABSTRACT}

Background and objectives: Colorectal cancer (CRC) is one of the leading causes of cancer-related mortality in the world. MicroRNAs (miRNAs) have potential as diagnostic biomarkers for various diseases including cancer. This study was undertaken to investigate expression of miR-2l before and after surgery in patients with hereditary CRC.

Methods: After collecting blood samples from 39 patients and 39 healthy controls, total RNA was extracted by the TRIzol method. Following cDNA synthesis, expression of miR21 in serum of subjects was evaluated using real-time PCR, along with two reference genes, let-7d and let-7g. The real-time expression results and $\mathrm{tt}$ values were collected and analyzed based on the $2-\Delta \Delta$ ct method.

Results: In spite of tumor removal, serum miR-2l expression levels was significantly higher in hereditary CRC patients compared with controls ( $\mathrm{P}=0.022)$.

Conclusion: Our results confirmed that samples from hereditary cases of CRC must not be included in experiments on the diagnostic potential of miRNAs.

Keywords: Colorectal cancer, Gene Expression, Real-Time Polymerase Chain Reaction. 


\section{INTRODUCTION}

Colorectal cancer (CRC) is characterized as a tumor growth on the inner lining of the rectum or colon $(1,2)$. It is the third most common cancer among both men and women (3). Growing westernized diet have led to an increase in the incidence and mortality of CRC (4). Indeed, CRC is complicated by the difficulty of early diagnosis, due to the lack of reliable cancer-specific diagnostic biomarkers (5). MicroRNAs (miRNAs) are a class of noncoding RNAs of nearly 22 bases in length that play crucial roles in both physiological and pathological processes. In addition, they are involved in cancer pathogenesis from initiation to metastasis, primarily through interaction with the 3' of target mRNA, which leads to post-transcriptional inhibition or mRNA degradation (6). In this respect, growing body of evidence have indicated that certain miRNAs released from tumor cells are chemically stable and can be detected in a broad range of body fluids, including plasma, which makes quick diagnosis feasible $(7,8)$. Therefore, miRNAs have emerged as promising novel biomarkers for CRC diagnosis. So far, hundreds of miRNAs have been reported to be associated with CRC progression and metastasis (9). A large number of studies have been performed to investigate the expression level of circulating miR-21 in blood samples of cancer patients and healthy individuals. However, results of the studies on the diagnostic value of circulating miR-21 have been inconsistent $(10,11)$. Here, we intended to investigate the expression level of mir-21 in Iranian patients with hereditary CRC and healthy individuals.

\section{MATERIALS AND METHODS}

A total of 39 whole blood samples were collected from CRC patients before and after surgical intervention at Qaem hospital in Mashhad, Iran. Age- and gender-matched healthy individuals (39 controls) with no history of cancer were also enrolled in thestudy. The study was performed between March 2018 and September 2019 in the Department of Biotechnology at Golestan University of Medical Sciences, Gorgan, Iran. Ethical approval was obtained from the Ethics Committee of Golestan University of Medical Sciences. In addition, an informed written consent form was obtained from all participants. First, sera were separated from the blood samples by centrifugation at 12,000 $\mathrm{g}$ for $10 \mathrm{~min}$ at $4{ }^{\circ} \mathrm{C}$. Then, miRNA extraction was carried out using RNx-PLUS kit (Sinagene Company) according to the manufacturer's protocol. The purity and quality of the extracted RNAs was checked and samples with absorbance of 1.8-2 at $260 / 280 \mathrm{~nm}$ were used. Moreover, the concentration of the extracted RNAs was assessed using a bio-photometer (Eppendorf, Germany), which produced an average concentration of $110 \mathrm{ng} / \mu \mathrm{l}$. Finally, real-time PCR was performed using specific primers for the miR-21. Internal control (let-7d and let-7g) was used due to the relativity of the method. For the real-time PCR reaction, $10 \mu 1$ SYBER Green master mix, $5 \mu \mathrm{l}$ of the synthetized cDNA and $4 \mu \mathrm{l}$ specific primers were mixed in a total volume of $20 \mu \mathrm{l}$. The reaction was carried out using the ABI 7300 model (Applied Biosystems) at optimal temperatures. Next, miR-21 expression level was calculated based on the $\triangle \Delta C T$ method. Data were analyzed using SPSS statistical software (version 22) and Graph Pad.

\section{RESULTS}

Based on the results, miR-21 was overexpressed in in postoperative serum samples from CRC patients who underwent curative surgery $(\mathrm{P}=0.022)$ (Table 1). The obtained $\mathrm{Ct}$ values from the real-time expression analysis indicated thatmiR-21 level increased almost 4.5-fold in sera of patients after tumor removal (Figure 1)

Table 1- Expression analysis of miR-21 after tumor removal

\begin{tabular}{|c|c|c|c|c|c|c|c|}
\hline Gene & Type & Reaction efficiency & Expression & Std.error & 95\% c.i. & $\mathbf{P}(\mathbf{h} 1)$ & Result \\
\hline$m i R-21$ & Target & 1.0 & 4.683 & $0.566-41.907$ & 0.096 - 378.272 & 0.022 & up \\
\hline let7-D & Reference & 1.0 & 0.772 & & & & \\
\hline $\operatorname{let} 7-G$ & Reference & 1.0 & 1.296 & & & & \\
\hline
\end{tabular}




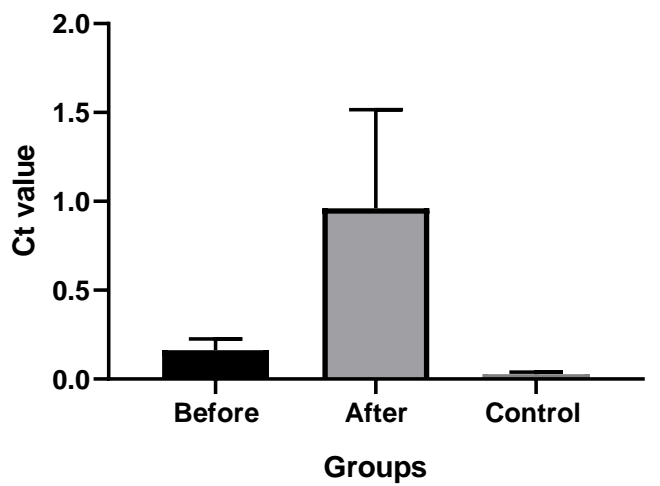

\section{DISCUSSION}

In this study, we aimed to explore the association between miRNA expression patterns and the diagnostic outcome of hereditary CRC. Since the beginning of the new century, the link between miRNAs and human diseases, particularly cancer, has been established. Although most miRNAs are intracellular, a large number of miRNAs have been detected outside cells in various body fluids. They are stable in cell culture supernatants and in many body fluids such as blood, urine, saliva and milk (12).

Results from recent studies indicate that circulating miRNAs could serve as potential diagnostic and prognostic markers in different types of cancers and other diseases. Additionally, early and timely diagnosis of cancer is one of the main objectives of today's cancer research projects worldwide. Thus, there has been a need for non-invasive biomarkers that would improve the early detection of different types of cancer including CRC $(13,14)$.

Furthermore, the expression pattern of miRNAs in the circulation could be relevant to the risk of disease progression in CRC.

\section{References}

1. Marley AR, Nan H. Epidemiology of colorectal cancer. International journal of molecular epidemiology and genetics. 2016; 7: 105-114. [PubMed] [Google Scholar]

2. Kolligs FT. Diagnostics and epidemiology of colorectal cancer. Visceral medicine. 2016; 32: 158-164. [DOI:10.1159/000446488] [PubMed] [Google Scholar]

3. Aran V, Victorino AP, Thuler LC, Ferreira CG . Colorectal cancer: epidemiology, disease mechanisms and interventions to reduce onset and mortality. Clinical Colorectal Cancer. 2016; 15: 195-203. [DOI:10.1016/j.clcc.2016.02.008] [PubMed] [Google Scholar]
However, inclusion criteria for evaluating miRNA expression from patients with hereditary cancer have long been considered to use patients without diabetes, metabolic diseases and hereditary cancer. Moreover, cases with history of medications, radiation therapy or even smoking have been excluded from the miRNA expression analysis studies (15).

\section{CONCLUSION}

Our study verified previous results concerning the exclusion of samples from patients with hereditary cancer when investigating diagnostic potential of miRNAs expression in cancer studies.

\section{ACKNOWLEDGEMENTS}

The authors would like to thank Deputy of Research and Technology of Golestan University of Medical Sciences and all those who participated in this study.

\section{CONFLICT OF INTEREST}

The authors declare that there is no conflict of interest.

4. Chen W, Zheng R, Baa de PD, Zhang S, Zeng H, Bray F, et al. Cancer statistics in China, 2015. CA: A Cancer Journal for Clinicians. 2016; 66: 115-132. [DOI:10.3322/caac.21338] [PubMed] [Google Scholar]

5. Hamada T, Nishihara R, Ogino S. Post-colonoscopy colorectal cancer: the key role of molecular pathological epidemiology. Translational gastroenterology and hepatology. $\quad 2017 ; \quad 2: \quad 9$. [DOI:10.21037/tgh.2017.01.05] [PubMed] [Google Scholar] 
6. Reddy KB. MicroRNA (miRNA) in cancer. Cancer cell international. 2015; 15: 38. [DOI:10.1186/s12935015-0185-1] [PubMed] [Google Scholar]

7. Sun Y, Liu Y, Cogdell D, Calin GA, Sun B, Kopetz S, et al. Examining plasma microRNA markers for colorectal cancer at different stages. Oncotarget. 2016; 7: 11434-11449. [DOI:10.18632/oncotarget.7196] [PubMed] [Google Scholar]

8. Wang S, Xiang J, Li Z, Lu S, Hu J, Gao X, et al. A plasma microRNA panel for early detection of colorectal cancer. International journal of cancer. 2015; 136(1): 152-161. [DOI:10.1002/ijc.28136] [PubMed] [Google Scholar]

9. Fang Z, Tang J, Bai Y, Lin H, You H, Jin H, et al. Plasma levels of microRNA-24, microRNA-320a, and microRNA-423-5p are potential biomarkers for colorectal carcinoma. Journal of experimental and clinical cancer research. 2015; 34: 86. [DOI:10.1186/s13046-015-0198-6] [PubMed] [Google Scholar]

10. Asaga S, Kuo C, Nguyen T, Terpenning M, Giuliano AE, Hoon DS. Direct serum assay for microRNA-21 concentrations in early and advanced breast cancer. Clinical chemistry. 2011; 57: 84-91. [DOI:10.1373/clinchem.2010.151845] [PubMed] [Google Scholar]

11. Kanaan Z, Rai SN, Eichenberger MR, Roberts H, Keskey B, Pan J, et al. Plasma MiR-21: a potential diagnostic marker of colorectal cancer. Annals of Surgery. 2012; 256: 544-51. [DOI:10.1097/SLA.0b013e318265bd6f] [PubMed] [Google Scholar]
12. Mitchell PS, Parkin RK, Kroh EM, Fritz BR, Wyman SK, Pogosova-Agadjanyan EL, et al. Circulating microRNAs as stable bloodbased markers for cancer detection. Proceedings of the national academy of sciences. U.S.A. 2008; 105(30): 10513-8. [DOI:10.1073/pnas.0804549105] [PubMed] [Google Scholar]

13. Chen X, Ba Y, Ma L, Cai X, Yin Y, Wang K, et al, et al. Characterization of microRNAs in serum: a novel class of biomarkers for diagnosis of cancer and other diseases. Cell Research. 2008; 18(10): 997-1006. [DOI:10.1038/cr.2008.282] [PubMed] [Google Scholar]

14. Gilad S, Meiri E, Yogev Y, Benjamin S, Lebanony $\mathrm{D}$, Yerushalmi N, et al. Serum microRNAs are promising novel biomarkers. PLoS ONE. 2008; 3: e3148. [DOI:10.1371/journal.pone.0003148] [PubMed] [Google Scholar]

15. Toiyama Y, Takahashi M, Hur K, Nagasaka T, Tanaka K, Inoue Y, et al. Serum miR-21 as a diagnostic and prognostic biomarker in colorectal cancer. Journal of the national cancer institute. 2013; 105(12): 849-59. [DOI:10.1093/jnci/djt101] [PubMed] [Google Scholar]

How to Cite:

Arefi M, Abdollahi A, Khosravi A, Moradi A, Aghaee-Bakhtiari SH, Javid N, Evazalipour M[Expression Analysis of Circulating miR-21 in Iranian Patients with Hereditary Colorectal Cancer]. mljgoums. 2021; 15(2): 1-4 DOI: 10.29252/mlj.15.2.1

(C) The authors 